\title{
ON RIEMANNIAN MANIFOLDS WITH POSITIVE WEIGHTED RICCI CURVATURE OF NEGATIVE EFFECTIVE DIMENSION
}

\author{
Cong Hung MAI \\ (Received 20 February 2018 and revised 10 July 2018)
}

\begin{abstract}
In this paper, we investigate complete Riemannian manifolds satisfying the lower weighted Ricci curvature bound $\operatorname{Ric}_{N} \geq K$ with $K>0$ for the negative effective dimension $N<0$. We analyze two one-dimensional examples of constant curvature $\operatorname{Ric}_{N} \equiv K$ with finite and infinite total volumes. We also discuss when the first non-zero eigenvalue of the Laplacian takes its minimum under the same condition $\operatorname{Ric}_{N} \geq K>0$, as a counterpart to the classical Obata rigidity theorem. Our main theorem shows that, if $N<-1$ and the minimum is attained, then the manifold splits off the real line as a warped product of hyperbolic nature.
\end{abstract}

\section{Introduction}

Riemannian manifolds of Ricci curvature bounded below are classical research subjects in comparison geometry and geometric analysis. Recently, diverse developments on the curvature-dimension condition in the sense of Lott, Sturm and Villani have shed new light on this theory. The curvature-dimension condition $\operatorname{CD}(K, N)$ is a synthetic notion of lower Ricci curvature bounds for metric measure spaces. The parameters $K$ and $N$ are usually regarded as 'a lower bound of the Ricci curvature' and 'an upper bound of the dimension', respectively. Thus $N$ is sometimes called the effective dimension. The roles of $K$ and $N$ are better understood when we consider a weighted Riemannian manifold $(M, g, m)$, a Riemannian manifold of dimension $n$ equipped with an arbitrary smooth measure $m$. Then the Ricci curvature is modified into the weighted Ricci curvature Ric $_{N}$ involving a parameter $N$, and $\mathrm{CD}(K, N)$ is equivalent to $\operatorname{Ric}_{N} \geq K$ (see [vRS, St1, St2, LV] as well as [Vi] for $N \in[n, \infty],[\mathbf{O h} 2, \mathbf{O h} 3]$ for $N \leq 0$, and also [Oh1] for the Finsler analogue).

For $N \in[n, \infty]$, the weighted Ricci curvature $\operatorname{Ric}_{N}$ (also called the Bakry-Émery-Ricci curvature) has been intensively studied by, for instance, Bakry and his collaborators in the framework of the $\Gamma$-calculus (see [BGL]). Recently it turned out that there is a rich theory also for $N \in(-\infty, 1]$, though this range seems strange due to the above interpretation of $N$ as an upper dimension bound. Among others, various Poincaré-type inequalities [KM], the curvature-dimension condition [Oh2, Oh3], isoperimetric inequalities [Mi, Kl, Oh3], and the splitting theorem [Wy] were studied for $N<0$ or $N \leq 1$.

2010 Mathematics Subject Classification: Primary 53C24.

Keywords: spectral gap; weighted Ricci curvature; negative effective dimensions.

(C) 2019 Faculty of Mathematics, Kyushu University 
The aim of this article is to contribute to the study of the structure of Riemannian manifolds with $\operatorname{Ric}_{N} \geq K$ for $K>0$ and $N<0$. In Section 3 we analyze two onedimensional examples $M_{1}, M_{2}$ of $\operatorname{Ric}_{N} \equiv K$ (appearing in [KM, Mi]) having different natures. The first example $M_{1}$ has finite volume and enjoys only the exponential concentration; in particular, $M_{1}$ does not satisfy the logarithmic Sobolev inequality. For $N<-1$, this space attains the minimum of the first non-zero eigenvalue of the (weighted) Laplacian (derived from the Bochner inequality, see Proposition 2.5). The second example $M_{2}$ has infinite volume and reveals the difficulty in obtaining a volume comparison under $\operatorname{Ric}_{N} \geq K$.

In Section 4, we investigate when the minimum of the first non-zero eigenvalue is attained under the condition $\operatorname{Ric}_{N} \geq K>0$ with $N<-1$, as a counterpart to the Obata rigidity theorem $[\mathbf{O b}]$. Our main theorem (Theorem 4.5) asserts that, when $\operatorname{dim} M \geq 2$, we have a warped product splitting $M \cong \mathbb{R} \times_{\cosh (\sqrt{K /(1-N)} t)} \Sigma^{n-1}$ of hyperbolic nature. Moreover, $\Sigma$ enjoys $\operatorname{Ric}_{N-1} \geq K(2-N) /(1-N)$. This warped product splitting should be compared with Cheng-Zhou's theorem in the case of $N=\infty$, where the sharp spectral gap forces the space to isometrically split off a one-dimensional Gaussian space (see $[\mathbf{C Z}]$ and Theorem 4.3 for details). Our proof considers the equality case in the Bochner inequality, which is related to the dimension-free version in $[\mathbf{C Z}]$ but requires a further discussion due to the fact that the Hessian of the eigenfunction does not vanish (Lemma 4.4). Therefore we have only the warped product rather than the isometric product in $[\mathbf{C Z}]$. We stress that in our setting of $N<0$, it is interesting to have a hyperbolic structure even when the curvature is positive.

\section{Preliminaries}

\subsection{Weighted Riemannian manifolds}

A weighted Riemannian manifold $(M, g, m)$ will be a pair of a complete, connected, boundaryless manifold $M$ equipped with a Riemannian metric $g$ and a measure $m=e^{-\psi}$ vol $_{g}$, where $\psi \in C^{\infty}(M)$ and $\operatorname{vol}_{g}$ is the standard volume measure on $(M, g)$. On $(M, g, m)$, we define the weighted Ricci curvature as follows.

Definition 2.1. (Weighted Ricci curvature) Given a unit vector $v \in U_{x} M$ and $N \in(-\infty, 0) \cup$ $[n, \infty]$, the weighted Ricci curvature $\operatorname{Ric}_{N}(v)$ is defined by:

(1) $\operatorname{Ric}_{N}(v):=\operatorname{Ric}_{g}(v)+\operatorname{Hess} \psi(v, v)-\langle\nabla \psi(x), v\rangle^{2} /(N-n) \quad$ for $\quad N \in(-\infty, 0) \cup$ $(n, \infty)$;

(2) $\operatorname{Ric}_{n}(v):=\operatorname{Ric}_{g}(v)+\operatorname{Hess} \psi(v, v)$ if $\langle\nabla \psi(x), v\rangle=0$, and $\operatorname{Ric}_{n}(v):=-\infty$ otherwise;

(3) $\operatorname{Ric}_{\infty}(v):=\operatorname{Ric}_{g}(v)+\operatorname{Hess} \psi(v, v)$,

where $n=\operatorname{dim} M$ and $\operatorname{Ric}_{g}$ denotes the Ricci curvature of $(M, g)$. The parameter $N$ is sometimes called the effective dimension. We also define $\operatorname{Ric}_{N}(c v):=c^{2} \operatorname{Ric}_{N}(v)$ for $c \geq 0$.

Note that if $\psi$ is constant then the weighted Ricci curvature coincides with $\operatorname{Ric}_{g}(v)$ for all $N$. When $\operatorname{Ric}_{N}(v) \geq K$ holds for some $K \in \mathbb{R}$ and all unit vectors $v \in T M$, we will write $\operatorname{Ric}_{N} \geq K$. By definition,

$$
\operatorname{Ric}_{n}(v) \leq \operatorname{Ric}_{N}(v) \leq \operatorname{Ric}_{\infty}(v) \leq \operatorname{Ric}_{N^{\prime}}(v)
$$


holds for $n \leq N<\infty$ and $-\infty<N^{\prime}<0$, and $\operatorname{Ric}_{N}(v)$ is non-decreasing in $N$ in the ranges $(-\infty, 0)$ and $[n, \infty]$.

Remark 2.2. The weighted Ricci curvature for $N \in[n, \infty]$ has been intensively and extensively investigated, see [Qi, BGL] for instance. The study for the negative effective dimension $N<0$ is more recent. One can find in [Mi, Kl, Oh3] the isoperimetric inequality, in $[\mathbf{W y}]$ the Cheeger-Gromoll type splitting theorem, and in [Oh2, Oh3] the curvaturedimension condition in this context.

We also define the weighted Laplacian with respect to $m$.

Definition 2.3. (Weighted Laplacian) The weighted Laplacian (also called the Witten Laplacian) of $u \in C^{\infty}(M)$ is defined as follows:

$$
\Delta_{m} u:=\Delta u-\langle\nabla u, \nabla \psi\rangle .
$$

Notice that the Green formula (the integration by parts formula)

$$
\int_{M} u \Delta_{m} v d m=-\int_{M}\langle\nabla u, \nabla v\rangle d m=\int_{M} v \Delta_{m} u d m
$$

holds provided $u$ or $v$ belongs to $C_{c}^{\infty}(M)$ (smooth functions with compact supports) or $H_{0}^{1}(M)$.

\subsection{Bochner inequality and eigenvalues of the Laplacian}

Recall that the Bochner-Weitzenböck formula associated with the weighted Ricci curvature $\operatorname{Ric}_{\infty}$ and the weighted Laplacian $\Delta_{m}$ holds as follows. For $u \in C^{\infty}(M)$, we have

$$
\Delta_{m}\left(\frac{|\nabla u|^{2}}{2}\right)-\left\langle\nabla \Delta_{m} u, \nabla u\right\rangle=\operatorname{Ric}_{\infty}(\nabla u)+\|\operatorname{Hess} u\|_{H S}^{2},
$$

where $\|\cdot\|_{H S}$ stands for the Hilbert-Schmidt norm (with respect to $g$ ). As a corollary we obtain the Bochner inequality for $N \in(-\infty, 0) \cup[n, \infty]$ (the case of negative effective dimension was studied independently in [KM, Oh2]). We give an outline of the proof for later use.

TheOREM 2.4. (Bochner inequality) For $N \in(-\infty, 0) \cup[n, \infty]$ and any $u \in C^{\infty}(M)$, we have

$$
\Delta_{m}\left(\frac{|\nabla u|^{2}}{2}\right)-\left\langle\nabla \Delta_{m} u, \nabla u\right\rangle \geq \operatorname{Ric}_{N}(\nabla u)+\frac{\left(\Delta_{m} u\right)^{2}}{N} .
$$

Proof. Fix $x \in M$ and let $B$ be the matrix representation of Hess $u(x)$ for some orthonormal basis. Then we find

$$
\| \text { Hess } u \|_{H S}^{2}=\operatorname{tr}\left(B^{2}\right) \geq \frac{(\operatorname{tr} B)^{2}}{n}=\frac{(\Delta u)^{2}}{n} .
$$

Putting $p=\Delta_{m} u$ and $q=\langle\nabla u, \nabla \psi\rangle$, we have

$$
\begin{aligned}
\frac{(\Delta u)^{2}}{n} & =\frac{(p+q)^{2}}{n}=\frac{p^{2}}{N}-\frac{q^{2}}{N-n}+\frac{N(N-n)}{n}\left(\frac{p}{N}+\frac{q}{N-n}\right)^{2} \\
& \geq \frac{p^{2}}{N}-\frac{q^{2}}{N-n}=\frac{\left(\Delta_{m} u\right)^{2}}{N}-\frac{\langle\nabla u, \nabla \psi\rangle^{2}}{N-n} .
\end{aligned}
$$

Combining these with the Bochner-Weitzenböck formula (1) we obtain the Bochner inequality. 
We remark that $N(N-n) \geq 0$ used in the proof fails for $N \in(0, n)$. The Bochner inequality has quite rich applications in geometric analysis. In this article we are interested in the first non-zero eigenvalue of the weighted Laplacian, a generalization of the Lichnerowicz inequality. The case of the negative effective dimension $N<0$ was studied in [KM, Oh2]. Here we give a proof for completeness.

Proposition 2.5. (First non-zero eigenvalue) Let $(M, g, m)$ be a complete weighted Riemannian manifold satisfying $\operatorname{Ric}_{N} \geq K$ for some $K>0$ and $N<0$, and assume $m(M)<$ $\infty$. Then the first non-zero eigenvalue of the non-negative operator $-\Delta_{m}$ is bounded from below by $K N /(N-1)$.

Proof. Let $u \in H_{0}^{1} \cap C^{\infty}(M)$ be a non-constant eigenfunction of $\Delta_{m}$ with $\Delta_{m} u=-\lambda u$, $\lambda>0$. We deduce from the Bochner inequality (Theorem 2.4) and the condition $\operatorname{Ric}_{N} \geq K$ that

$$
\Delta_{m}\left(\frac{|\nabla u|^{2}}{2}\right) \geq\left\langle\nabla \Delta_{m} u, \nabla u\right\rangle+K|\nabla u|^{2}+\frac{\left(\Delta_{m} u\right)^{2}}{N} .
$$

Since constant functions belong to $H_{0}^{1}(M)$ thanks to the hypothesis $m(M)<\infty$, the integration of the above inequality yields

$$
0 \geq\left(\frac{1}{N}-1\right) \int_{M}\left(\Delta_{m} u\right)^{2} d m+K \int_{M}|\nabla u|^{2} d m .
$$

We again use the integration by parts to see

$$
\int_{M}\left(\Delta_{m} u\right)^{2} d m=-\lambda \int_{M} u \Delta_{m} u d m=\lambda \int_{M}|\nabla u|^{2} d m
$$

Therefore we have

$$
\frac{1-N}{N} \lambda+K \leq 0
$$

which shows the claim $\lambda \geq K N /(N-1)$.

It was observed in [KM, §3.2] and [Mi, Theorem 6.1] that the estimate $\lambda \geq K N /(N-1)$ is sharp for $N \in(-\infty,-1]$ and, somewhat surprisingly, not sharp for $N<0$ close to 0 . See Section 4 for a further discussion, where we discuss the rigidity for the constant $K N /(N-1)$ in the case $N<-1$. The first non-zero eigenvalue of the weighted Laplacian is related to the concentration of measures. When $m(M)=1$, we define the concentration function of $(M, g, m)$ by

$$
\alpha(r):=\sup \{1-m(B(A, r)) \mid A \subset M: \text { Borel, } m(A) \geq 1 / 2\} .
$$

See [Le, Theorem 3.1] for the following corollary.

COROLlaRY 2.6. (Exponential concentration) Let $(M, g, m)$ be a compact weighted Riemannian manifold satisfying $\operatorname{Ric}_{N} \geq K$ for some $K>0$ and $N<0$, and assume $m(M)$ $=1$. Then $(M, g, m)$ satisfies the exponential concentration $\alpha(r) \leq e^{-\sqrt{K N /(N-1)} r / 3}$. 


\section{Two examples of one-dimensional spaces of constant curvature}

In this section we analyze two examples of one-dimensional spaces such that $\operatorname{Ric}_{N} \equiv K$ for some $K>0$ and $N<0$. These examples will be helpful to understand the general picture of the spaces satisfying $\operatorname{Ric}_{N} \geq K>0$. In particular, we shall give negative answers to some naive guesses.

The first example is the following.

Example 3.1. For $K>0$ and $N<0$, the space

$$
M_{1}:=\left(\mathbb{R},|\cdot|, \cosh \left(\sqrt{\frac{K}{1-N}} x\right)^{N-1} d x\right),
$$

where $|\cdot|$ denotes the canonical distance structure, satisfies $\operatorname{Ric}_{N} \equiv K$.

This is a model space in Milman's isoperimetric inequality (see Case 1 of [Mi, Corollary 1.4]). Notice that the total volume is finite since

$$
\begin{aligned}
\int_{\mathbb{R}} \cosh \left(\sqrt{\frac{K}{1-N} x}\right)^{N-1} d x & \leq 2 \int_{0}^{\infty}\left(e^{\sqrt{K /(1-N)} x}\right)^{N-1} d x \\
& =2 \int_{0}^{\infty} e^{-\sqrt{K(1-N)} x} d x<\infty
\end{aligned}
$$

In order to see the curvature identity, we observe that the weight function is given by

$$
\psi(x)=(1-N) \log \left(\cosh \sqrt{\frac{K}{1-N} x}\right),
$$

and

$$
\begin{aligned}
& \psi^{\prime}(x)=\sqrt{K(1-N)} \tanh \left(\sqrt{\frac{K}{1-N}} x\right), \\
& \psi^{\prime \prime}(x)=K-K \tanh \left(\sqrt{\frac{K}{1-N} x}\right)^{2} .
\end{aligned}
$$

Since the Ricci curvature vanishes in the one-dimensional case, we have the curvature identity

$$
\begin{aligned}
\operatorname{Ric}_{N}\left(\left.\frac{\partial}{\partial x}\right|_{x}\right) & =\psi^{\prime \prime}(x)+\frac{\psi^{\prime}(x)^{2}}{1-N} \\
& =K-K \tanh \left(\sqrt{\frac{K}{1-N}} x\right)^{2}+\frac{K(1-N)}{1-N} \tanh \left(\sqrt{\frac{K}{1-N}} x\right)^{2} \\
& =K
\end{aligned}
$$

In $[\mathbf{K M}]$ it was observed that this space attains the minimum value of the first non-zero eigenvalue in respect of Proposition 2.5.

Lemma 3.2. (Minimal first non-zero eigenvalue) For $N<-1$, the first non-zero eigenvalue of $-\Delta_{m}$ in the space $M_{1}$ in Example 3.1 coincides with $K N /(N-1)$. 
Proof. We shall show that the function

$$
u(x)=\sinh \left(\sqrt{\frac{K}{1-N}} x\right)
$$

gives the minimal first non-zero eigenvalue. Notice that $u \in L^{2}\left(M_{1}\right)$ by the assumption $N<-1$, and

$$
u^{\prime}(x)=\sqrt{\frac{K}{1-N}} \cosh \left(\sqrt{\frac{K}{1-N}} x\right), \quad u^{\prime \prime}(x)=\frac{K}{1-N} u .
$$

Thus the weighted Laplacian of $u$ is calculated as

$$
\begin{aligned}
\Delta_{m} u(x) & =u^{\prime \prime}(x)-u^{\prime}(x) \psi^{\prime}(x)=\frac{K}{1-N} u(x)-K \sinh \left(\sqrt{\frac{K}{1-N}} x\right) \\
& =\frac{K}{1-N} u(x)-K u(x)=-\frac{K N}{N-1} u(x) .
\end{aligned}
$$

Remark 3.3. (Failure of log-Sobolev inequality) For $N \in[n, \infty]$, the bound $\operatorname{Ric}_{N} \geq K>0$ is known to imply the logarithmic Sobolev inequality (see, for example, [BGL] for proofs based on the $\Gamma$-calculus):

$$
\int_{\{f>0\}} f \log f d m \leq \frac{N-1}{2 K N} \int_{\{f>0\}} \frac{|\nabla f|^{2}}{f} d m
$$

for non-negative functions $f \in H^{1}(M)$ with $\int_{M} f d m=1$. It is well known that the logarithmic Sobolev inequality implies the normal concentration, see [Le, Theorem 5.3]. One might expect that the logarithmic Sobolev inequality (2) has a counterpart for $N<0$ similarly to the spectral gap. This is, however, not the case since $M_{1}$ (normalized as $m\left(M_{1}\right)=1$ ) given in Example 3.1 enjoys only the exponential concentration (consider $A=[0, \infty)$ ). See [Mi, Proposition 6.4] for a detailed estimate of the concentration function.

Our second example is as follows.

Example 3.4. For $K>0$ and $N<0$, the space

$$
M_{2}:=\left(\mathbb{R},|\cdot|, e^{-\sqrt{K(1-N)} x} d x\right)
$$

satisfies $\operatorname{Ric}_{N} \equiv K$.

This space also appeared in Case 2 of [Mi, Corollary 1.4]. The weight function is the linear function $\psi(x)=\sqrt{K(1-N)} x$ and the curvature identity is straightforward:

$$
\operatorname{Ric}_{N}\left(\left.\frac{\partial}{\partial x}\right|_{x}\right)=\frac{\psi^{\prime}(x)^{2}}{1-N}=K .
$$

We stress that $M_{2}$ has very different natures from $M_{1}$ in Example 3.1. Recall that, when $N=\infty, \operatorname{Ric}_{\infty} \geq K>0$ implies that the measure has Gaussian decay and the total volume is finite [St1, Theorem 4.26]. From Example 3.1 one may expect that $\operatorname{Ric}_{N} \geq K>0$ for $N<0$ still implies the exponential decay; however, it is not the case by the second example $M_{2}$ above. We refer to [Sa2] for a related work on weighted Riemannian manifolds 
with boundaries, where a volume comparison for regions spreading from the boundary was established for $N \leq 1$.

We also remark that, different from the case of $N=\infty$, taking products will destroy the curvature bound for $N<0$ due to the nonlinearity of the term $\langle\nabla \psi, v\rangle^{2}$ in $\psi$ in the definition of $\operatorname{Ric}_{N}(v)$. For instance,

$$
\left(\mathbb{R}^{n},|\cdot|, e^{-x^{1}-x^{2}-\cdots-x^{n}} d x^{1} d x^{2} \cdots d x^{n}\right)
$$

satisfies only $\operatorname{Ric}_{N} \geq 0$ (consider $v$ in the kernel of $d \psi$ ).

\section{First non-zero eigenvalue of the Laplacian}

As we saw in Proposition 2.5, the first non-zero eigenvalue of $-\Delta_{m}$ on a weighted Riemannian manifold satisfying $\operatorname{Ric}_{N} \geq K>0$ is bounded from below by $K N /(N-1)$ (or $K$ if $N=\infty$ ), which is sharp for $N \leq-1$. In this section we consider the rigidity problem when the minimum value is attained. In the unweighted $(N=n)$ case, the classical Obata theorem $[\mathbf{O b}]$ asserts the following.

THEOREM 4.1. (Obata rigidity theorem) Let $(M, g)$ be an $n$-dimensional Riemannian manifold with $\operatorname{Ric}_{g} \geq K>0$. Then the first non-zero eigenvalue of $-\Delta$ coincides with $K n /(n-1)$ if and only if $M$ is isometric to the $n$-dimensional sphere $\mathbb{S}^{n}$ with radius $\sqrt{(n-1) / K}$.

The case of $N \in(n, \infty)$ turns out void as follows.

THEOREM 4.2. (Ketterer, Kuwada) Let $(M, g, m)$ be a weighted Riemannian manifold of dimension $n$ with $\operatorname{Ric}_{N} \geq K>0$ for some $N \in[n, \infty)$. Then the first non-zero eigenvalue of $-\Delta_{m}$ coincides with $K N /(N-1)$ if and only if $N=n, m=c \operatorname{vol}_{g}$ for some constant $c>0$ and $M$ is isometric to the $n$-dimensional sphere $\mathbb{S}^{n}$ with radius $\sqrt{(n-1) / K}$.

Precisely, it is shown in $[\mathbf{K e}]$ in the general framework of RCD-spaces (metric measure spaces satisfying the Riemannian curvature-dimension condition) that the existence of an eigenfunction for the eigenvalue $K N /(N-1)$ implies the maximal diameter $\operatorname{diam}(M)=$ $\pi \sqrt{(N-1) / K}$ in the Bonnet-Myers type theorem. This is, however, achieved only when $N=n$ by Kuwada's result in [Ku]. If we admit singularities, then the sharp spectral gap is attained for spherical suspensions as discussed in [Ke] for RCD-spaces (see also [KM, §3.2]).

Next, in the case of $N=\infty$, an interesting rigidity theorem was established in [CZ].

THEOREM 4.3. (Cheng-Zhou) Let $(M, g, m)$ be an n-dimensional weighted Riemannian manifold with $\operatorname{Ric}_{\infty} \geq K>0$. If the first non-zero eigenvalue of $-\Delta_{m}$ coincides with $K$, then $M$ is isometric to the product space $\Sigma^{n-1} \times \mathbb{R}$ as weighted Riemannian manifolds, where $\Sigma^{n-1}$ is an $(n-1)$-dimensional manifold with $\operatorname{Ric}_{\infty} \geq K$ and $\mathbb{R}$ is equipped with the Gaussian measure $e^{-K x^{2} / 2} d x$.

The proof of Theorem 4.3 has a certain similarity with the Cheeger-Gromoll splitting theorem. The role of the Busemann function in [CG] is replaced with the eigenfunction in [CZ]. Theorem 4.3 was recently generalized to RCD-spaces in [GKKO].

Now we consider the case of $N<0$. We begin with an important equation for an eigenfunction. 
LEMMA 4.4. Let $(M, g, m)$ be a complete weighted Riemannian manifold satisfying $\operatorname{Ric}_{N} \geq$ $K$ for some $N<0$ and $K>0$, and $m(M)<\infty$. Suppose that the first non-zero eigenvalue of $-\Delta_{m}$ coincides with $K N /(N-1)$. Then the eigenfunction u of $K N /(N-1)$ necessarily satisfies

$$
\text { Hess } u=-\frac{K u}{N-1} g
$$

as operators $T M \times T M \longrightarrow \mathbb{R}$.

Proof. Let $u$ be an eigenfunction of the eigenvalue $K N /(N-1)$, namely

$$
\Delta_{m} u=-\frac{K N}{N-1} u .
$$

Tracing back to the proof of the lower bound of the first non-zero eigenvalue, the Bochner inequality for $u$ necessarily becomes an equality. Thus we find, from the proof of Theorem 2.4, that

$$
\begin{aligned}
& \text { Hess } u=f \cdot g \quad \text { for some function } f: M \longrightarrow \mathbb{R} ; \\
& \frac{\Delta_{m} u}{N}+\frac{\langle\nabla u, \nabla \psi\rangle}{N-n} \equiv 0 .
\end{aligned}
$$

On the one hand, we observe from the former equation (4) that $\Delta u=n f$. On the other hand, the latter equation (5) yields

$$
\Delta u=\Delta_{m} u+\langle\nabla u, \nabla \psi\rangle=\frac{n}{N} \Delta_{m} u=-\frac{K n}{N-1} u .
$$

Therefore we have $f=-K u /(N-1)$ and complete the proof.

THEOREM 4.5. Let $(M, g, m)$ be a complete weighted Riemannian manifold of dimension $n$ satisfying $\operatorname{Ric}_{N} \geq K$ for some $N<-1$ and $K>0$, and $m(M)<\infty$. Assume that the first non-zero eigenvalue of $-\Delta_{m}$ coincides with $K N /(N-1)$.

(i) If $n \geq 2$, then $M$ is isometric to the warped product

$$
\mathbb{R} \times \cosh (\sqrt{K /(1-N)} t) \Sigma=\left(\mathbb{R} \times \Sigma, d t^{2}+\cosh ^{2}\left(\sqrt{\frac{K}{1-N} t}\right) \cdot g_{\Sigma}\right)
$$

and the measure $m$ is written through the isometry as

$$
m(d t d x)=\cosh ^{N-1}\left(\sqrt{\frac{K}{1-N} t}\right) d t m_{\Sigma}(d x),
$$

where $\left(\Sigma, g_{\Sigma}, m_{\Sigma}\right)$ is an $(n-1)$-dimensional weighted Riemannian manifold satisfying $\operatorname{Ric}_{N-1} \geq K(2-N) /(1-N)$.

(ii) If $n=1$, then $(M, g, m)$ is isometric to the model space $M_{1}$ in Example 3.1 up to a constant multiplication of the measure.

Proof. (i) By rescaling the Riemannian metric, we can assume that $K=1-N$. Let $u \in$ $H_{0}^{1} \cap C^{\infty}(M)$ be an eigenfunction satisfying $\Delta_{m} u=N u$. Along any geodesic $\gamma: I \longrightarrow M$ for an interval $I \subset \mathbb{R}$, it follows from Lemma 4.4 that $(u \circ \gamma)^{\prime \prime}=(u \circ \gamma)$. This implies that

$$
u \circ \gamma(t)=u \circ \gamma(0) \cdot \cosh t+(u \circ \gamma)^{\prime}(0) \cdot \sinh t .
$$


Put $\Sigma:=u^{-1}(0)$ (which is non-empty since $\int_{M} u d m=0$ ). We deduce from (6) that, for $x, y \in \Sigma$, every geodesic connecting them is included in $\Sigma$. Hence $\Sigma$ is totally geodesic and the mean curvature of $\Sigma$ is identically zero. Lemma 4.4 also shows that, for any smooth vector field $V$ along a geodesic $\gamma$ included in $\Sigma$,

$$
\left\langle\nabla_{\dot{\gamma}}(\nabla u \circ \gamma), V\right\rangle=\operatorname{Hess} u(\dot{\gamma}, V)=u \cdot\langle\dot{\gamma}, V\rangle \equiv 0 .
$$

Hence $\nabla u \circ \gamma$ is a parallel vector field and $|\nabla u|$ is constant on $\Sigma$. We will normalize $u$ so as to satisfy $|\nabla u| \equiv 1$ on $\Sigma$.

Fix $x \in \Sigma$ and consider the geodesic $\gamma_{x}:[0, \infty) \longrightarrow M$ such that $\dot{\gamma}_{x}(0)=\nabla u(x)$. Then (6) and our normalization $|\nabla u|(x)=1$ yield

$$
\left(u \circ \gamma_{x}\right)(t)=\sinh t, \quad t \in \mathbb{R} .
$$

For any unit speed geodesic $\eta:[0, s] \longrightarrow M$ from $x$ to $\gamma_{x}(t),(7)$ and (6) imply $\sinh t=$ $u(\eta(s)) \leq \sinh s$. Since $\sinh t$ is monotone increasing, we have $s \geq t$ and $\gamma_{x}$ is globally minimizing. Denoting the distance function from $\Sigma$ by $\rho_{\Sigma}$, we obtain from (7) that $\rho_{\Sigma}=$ $|\operatorname{arcsinh}(u)|$ and, on $u^{-1}((0, \infty))$,

$$
\Delta u=\Delta\left(\sinh \rho_{\Sigma}\right)=\cosh \rho_{\Sigma} \cdot \Delta \rho_{\Sigma}+\sinh \rho_{\Sigma} \cdot\left|\nabla \rho_{\Sigma}\right|^{2} .
$$

Since $\left|\nabla \rho_{\Sigma}\right|=1$ and $\Delta u=n u=n \sinh \rho_{\Sigma}$, we have for $t \geq 0$

$$
\Delta \rho_{\Sigma}\left(\gamma_{x}(t)\right)=(n-1) \tanh \rho_{\Sigma}\left(\gamma_{x}(t)\right)=(n-1) \tanh t .
$$

We shall compare the Laplacian comparison inequality (8) with the (unweighted) Ricci curvature along $\gamma_{x}$. Using (7) and (5), we have

$$
\begin{aligned}
\left(\psi \circ \gamma_{x}\right)^{\prime}(t) & =\left\langle\nabla \psi \circ \gamma_{x}, \dot{\gamma}_{x}\right\rangle(t)=\frac{1}{\cosh t}\langle\nabla \psi, \nabla u\rangle\left(\gamma_{x}(t)\right) \\
& =\frac{n-N}{\cosh t} u\left(\gamma_{x}(t)\right)=(n-N) \tanh t .
\end{aligned}
$$

Hence

$$
\psi \circ \gamma_{x}(t)=(n-N) \log (\cosh t)+\psi(x)
$$

and

$$
\begin{aligned}
\operatorname{Ric}\left(\dot{\gamma}_{x}(t)\right) & =\operatorname{Ric}_{N}\left(\dot{\gamma}_{x}(t)\right)-\left(\psi \circ \gamma_{x}\right)^{\prime \prime}(t)-\frac{\left(\psi \circ \gamma_{x}\right)^{\prime}(t)^{2}}{n-N} \\
& =\operatorname{Ric}_{N}\left(\dot{\gamma}_{x}(t)\right)-\frac{n-N}{\cosh ^{2} t}-(n-N) \tanh ^{2} t \\
& =\operatorname{Ric}_{N}\left(\dot{\gamma}_{x}(t)\right)-(n-N) \geq 1-n .
\end{aligned}
$$

This curvature bound together with the minimality of $\Sigma$ implies that

$$
\Delta \rho_{\Sigma}\left(\gamma_{x}(t)\right) \leq(n-1) \tanh t
$$

by [Ka, Corollary 2.44] (see also [HK] and [Sa1, Theorem 4.3]). Then, since we have equality (8), the rigidity theorem for the Laplacian comparison shows that $u^{-1}([0, \infty))$ is 
isometric to the warped product

$$
[0, \infty) \times_{\cosh } \Sigma:=\left([0, \infty) \times \Sigma, d t^{2}+\cosh ^{2} t \cdot g_{\Sigma}\right),
$$

where $g_{\Sigma}$ is the Riemannian metric of $\Sigma$ induced from $g$ of $M$ (see the proof of [Sa1, Theorem 1.8] for details). Here we give a sketch of the proof for the isometry along [Sa1].

For a regular point $x \in \Sigma$ of $u$, let $T_{x}^{\perp} \Sigma$ be the orthogonal complement of $T_{x} \Sigma$. Choose an orthonormal basis $\left\{e_{x, i}\right\}_{i=1}^{n-1}$ of $T_{x} \Sigma$ and for $i=1, \ldots, n-1$, let $Y_{x, i}$ be the $\Sigma$-Jacobi field along $\gamma_{x}$ such that $Y(0)=e_{x, i}$ and $Y_{x, i}^{\prime}(0)=-A_{\nabla u(x)} e_{x, i}$, where $A_{v}: T_{x} \Sigma \longrightarrow T_{x} \Sigma$ is the shape operator of the tangent vector $v$. Since the mean curvature on $\Sigma$ is 0 and $\operatorname{Ric}_{N}\left(\dot{\gamma}_{x}(t)\right) \geq 1-n$, we have $\Delta \rho_{\Sigma}\left(\gamma_{x}(t)\right) \leq(n-1)$ tanh $t$ as in (10) and equality holds if and only if $Y_{x, i}(t)=\cosh (t) E_{x, i}(t)$ where $E_{x, i}$ are the parallel vector fields along $\gamma_{x}$ with $E_{x, i}(0)=e_{x, i}$. Then the map $\phi:[0, \infty) \times \Sigma \longrightarrow M$ defined by $\phi(t, x):=\gamma_{x}(t)$ provides the desired isometry between $[0, \infty) \times \cosh \Sigma$ and $M$.

By a similar discussion on $u^{-1}((-\infty, 0])$ we can extend the isometry to

$$
M \cong \mathbb{R} \times \cosh \Sigma=\left(\mathbb{R} \times \Sigma, d t^{2}+\cosh ^{2} t \cdot g_{\Sigma}\right) .
$$

Concerning the splitting of the measure, let $\Sigma$ be equipped with the measure $m_{\Sigma}:=$ $e^{-\left.\psi\right|_{\Sigma}} \operatorname{vol}_{g_{\Sigma}}$. By (9) and the structure of the warped product, we see that the measure $m$ is written through the isometry as

$$
\begin{aligned}
m(d t d x) & =\cosh ^{N-n} t \cdot e^{-\psi(x)} \cdot\left(\cosh ^{n-1} t d t \operatorname{vol}_{g_{\Sigma}}(d x)\right) \\
& =\cosh ^{N-1} t d t m_{\Sigma}(d x) .
\end{aligned}
$$

With this expression one can see that the eigenfunction $u$ is in $L^{2}(M)$ when $N<-1$ (but not in $L^{2}(M)$ for $N \in[-1,0)$ ).

Finally, for any unit vector $v \in T_{x} \Sigma$ at $x \in \Sigma$, the sectional curvature of the plane $\dot{\gamma}_{x}(0) \wedge v$ coincides with -1 (see [ON, Proposition 42(2) in §7]). Therefore we have, on the weighted Riemannian manifold $\left(\Sigma, g_{\Sigma}, m_{\Sigma}\right)$,

$$
\begin{aligned}
\operatorname{Ric}_{N-1}^{\Sigma}(v) & =\operatorname{Ric}^{\Sigma}(v)+\operatorname{Hess}^{\Sigma} \psi(v, v)-\frac{\left\langle\nabla^{\Sigma} \psi, v\right\rangle^{2}}{(N-1)-(n-1)} \\
& =\operatorname{Ric}^{M}(v)+1+\operatorname{Hess}^{M} \psi(v, v)-\frac{\left\langle\nabla^{M} \psi, v\right\rangle^{2}}{N-n} \\
& =\operatorname{Ric}_{N}^{M}(v)+1 \geq 2-N .
\end{aligned}
$$

(ii) Fix an eigenfunction $u$ of the eigenvalue $K N /(N-1)$, take $x \in M$ with $u(x)=0$ and choose a unit speed geodesic $\gamma: \mathbb{R} \longrightarrow M$ with $\gamma(0)=x$. It follows from Lemma 4.4 that

$$
u \circ \gamma(t)=(u \circ \gamma)^{\prime}(0) \cdot \sinh \left(\sqrt{\frac{K}{1-N}} t\right) .
$$

Since $u$ is not constant, we have $(u \circ \gamma)^{\prime}(0) \neq 0$ and hence $u$ is injective. Therefore $M$ is isometric to $\mathbb{R}$ and we will identify them via $\gamma$ as $\gamma(t)=t$.

Denote by $\psi: \mathbb{R} \longrightarrow \mathbb{R}$ the weight function, namely $m=e^{-\psi} d t$. Then it follows from

$$
-\frac{K N}{N-1} u=\Delta_{m} u=u^{\prime \prime}-u^{\prime} \psi^{\prime}
$$


that

$$
\begin{aligned}
\psi^{\prime}(t) & =\frac{1}{u^{\prime}(t)}\left(u^{\prime \prime}(t)+\frac{K N}{N-1} u(t)\right) \\
& =\sqrt{\frac{1-N}{K}}\left(\frac{K}{1-N}+\frac{K N}{N-1}\right) \tanh \left(\sqrt{\frac{K}{1-N}} t\right) \\
& =\sqrt{K(1-N)} \tanh \left(\sqrt{\frac{K}{1-N}} t\right) .
\end{aligned}
$$

Integrating this we have

$$
\psi(t)=(1-N) \log \left(\cosh \left(\sqrt{\frac{K}{1-N}} t\right)\right)+\psi(0),
$$

which implies $m=e^{-\psi(0)} \cosh (\sqrt{K /(1-N)} t)^{N-1} d t$ as desired.

The following corollary is a byproduct of the proof above (see [KM, Mi] for the onedimensional case).

COROLlary 4.6. Let $(M, g, m)$ be a complete weighted Riemannian manifold of dimension $n$ satisfying $\operatorname{Ric}_{N} \geq K$ for some $N \in[-1,0)$ and $K>0$, and $m(M)<\infty$. Then $K N /(N-1)$ cannot be an eigenvalue of $-\Delta_{m}$.

Remark 4.7. The curvature bound of $\Sigma$ implies the estimate of the first non-zero eigenvalue $\lambda_{1}(\Sigma)$ as

$$
\lambda_{1}(\Sigma) \geq \frac{K(2-N)}{1-N} \frac{N-1}{N-2}=K .
$$

This is better than the estimate $\lambda_{1}(M) \geq K N /(N-1)$ for $M$.

We close the article with a concrete example of the splitting phenomenon described in Theorem 4.5(i) (with $K=1-N$ ).

Example 4.8. Let $\left(\mathbb{H}^{2}, g\right)$ be the hyperbolic plane of constant sectional curvature -1 . There are smooth functions $u$ and $\psi$ satisfying

$$
\Delta_{m} u=N u, \quad \operatorname{Ric}_{N} \equiv 1-N,
$$

where $m=e^{-\psi} \operatorname{vol}_{g}$.

Let us give the precise expressions of $u$ and $\psi$ in the upper half-plane model:

$$
\left(\mathbb{H}^{2}, g\right)=\left(\mathbb{R} \times(0, \infty), \frac{d x^{2}+d y^{2}}{y^{2}}\right) .
$$

By the consideration in the proof of Theorem 4.5(i), we can expect that the function $u$ is written by using the distance function from the $y$-axis $\left(\Sigma=u^{-1}(0)\right.$ coincides with the 
$y$-axis). One can explicitly calculate it as

$$
\begin{aligned}
u(x, y) & = \pm \sinh \left(d\left((x, y),\left(0, \sqrt{x^{2}+y^{2}}\right)\right)\right) \\
& = \pm \sinh \left(\operatorname{arccosh}\left(1+\frac{x^{2}+\left(y-\sqrt{x^{2}+y^{2}}\right)^{2}}{2 y \sqrt{x^{2}+y^{2}}}\right)\right) \\
& = \pm \sqrt{\frac{x^{2}+y^{2}}{y^{2}}-1}=\frac{x}{y},
\end{aligned}
$$

where we choose the sign + for $x>0$ and - for $x<0$, and we used the equation $\sinh (\operatorname{arccosh} t)=\sqrt{t^{2}-1}$ for $t \geq 0$. The Christoffel symbols are readily calculated as

$$
\Gamma_{11}^{1}=\Gamma_{22}^{1}=\Gamma_{12}^{2} \equiv 0, \quad \Gamma_{12}^{1}(x, y)=\Gamma_{22}^{2}(x, y)=-\frac{1}{y}, \quad \Gamma_{11}^{2}(x, y)=\frac{1}{y} .
$$

Using these we find

$$
\begin{aligned}
(\operatorname{Hess} u)_{(x, y)} & =\left(\begin{array}{cc}
0 & -y^{-2} \\
-y^{-2} & 2 x y^{-3}
\end{array}\right)-\frac{1}{y}\left(\begin{array}{cc}
0 & -y^{-1} \\
-y^{-1} & 0
\end{array}\right)+\frac{x}{y^{2}}\left(\begin{array}{cc}
y^{-1} & 0 \\
0 & -y^{-1}
\end{array}\right) \\
& =\frac{x}{y} \cdot \frac{1}{y^{2}}\left(\begin{array}{ll}
1 & 0 \\
0 & 1
\end{array}\right)=u(x, y) \cdot g_{(x, y)} .
\end{aligned}
$$

Next we consider the weight function. Let

$$
\begin{aligned}
\psi_{1}(x, y): & =(2-N) \log \left[\cosh \left(d\left((x, y),\left(0, \sqrt{x^{2}+y^{2}}\right)\right)\right)\right] \\
& =(2-N) \log \left(\frac{\sqrt{x^{2}+y^{2}}}{y}\right),
\end{aligned}
$$

and

$$
\psi_{2}(x, y):=-(2-N) \log \left(\sqrt{x^{2}+y^{2}}\right) .
$$

Notice that $\psi_{1}$ naturally appears in respect of (5), and $\psi_{2}$ is employed to improve the convexity in the directions perpendicular to $\nabla u$. Indeed, we have $\left\langle\nabla u, \nabla \psi_{2}\right\rangle=0$ and

$$
\begin{aligned}
\left\langle\nabla u, \nabla \psi_{1}\right\rangle(x, y) & =(2-N) y^{2}\left(\frac{1}{y} \cdot \frac{x}{x^{2}+y^{2}}-\frac{x}{y^{2}} \cdot\left(\frac{y}{x^{2}+y^{2}}-\frac{1}{y}\right)\right) \\
& =(2-N) \frac{x}{y}=(2-N) u(x, y) .
\end{aligned}
$$

Our weighted function will be $\psi=\psi_{1}+\psi_{2}$, namely

$$
\psi(x, y)=-(2-N) \log y .
$$

The above calculations yield

$$
\Delta_{m} u=\Delta u-\langle\nabla u, \nabla \psi\rangle=2 u-(2-N) u=N u .
$$

We finally calculate $\operatorname{Ric}_{N}$ of this example. To this end, we observe that

$$
\frac{(\operatorname{Hess} \psi)_{(x, y)}}{2-N}=\left(\begin{array}{cc}
y^{-2} & 0 \\
0 & 0
\end{array}\right), \quad \frac{(d \psi \otimes d \psi)_{(x, y)}}{(2-N)^{2}}=\left(\begin{array}{cc}
0 & 0 \\
0 & y^{-2}
\end{array}\right) \text {. }
$$


Therefore we obtain

$$
\begin{aligned}
\left(\operatorname{Ric}_{N}\right)_{(x, y)} & =-\frac{1}{y^{2}}\left(\begin{array}{ll}
1 & 0 \\
0 & 1
\end{array}\right)+(\operatorname{Hess} \psi)_{(x, y)}+\frac{(d \psi \otimes d \psi)_{(x, y)}}{2-N} \\
& =\frac{1-N}{y^{2}}\left(\begin{array}{ll}
1 & 0 \\
0 & 1
\end{array}\right) .
\end{aligned}
$$

Acknowledgements. I would like to thank my supervisor, Professor Shin-ichi Ohta, for the kind guidance, encouragement and advice he has provided throughout my time working on this paper. I also would like to thank Professor Emanuel Milman for valuable comments on the earlier version of the paper, especially on the case of $N \in[-1,0)$.

\section{REFERENCES}

[BGL] D. Bakry, I. Gentil and M. Ledoux. Analysis and Geometry of Markov Diffusion Operators. Springer, Cham, 2014.

[CG] J. Cheeger and D. Gromoll. The splitting theorem for manifolds of non-negative Ricci curvature. J. Differential Geometry 6 (1971/72), 119-128.

[CZ] X. Cheng and D. Zhou. Eigenvalues of the drifted Laplacian on complete metric measure spaces. Commun. Contemp. Math. 19 (2017), 1650001, 17pp.

[GKKO] N. Gigli, C. Ketterer, K. Kuwada and S. Ohta. Rigidity for the spectral gap on $\operatorname{RCD}(K, \infty)$-spaces. Preprint, 2017, arXiv:1709.04017.

[HK] E. Heintze and H. Karcher. A general comparison theorem with applications to volume estimates for submanifolds. Ann. Sci. Éc. Norm. Supér. 11 (1978), 451-470.

[Ka] A. Kasue. A Laplacian comparison theorem and function theoretic properties of a complete Riemannian manifold. Japan J. Math. 8(2) (1982), 309-341.

[Ke] C. Ketterer. Obata's rigidity theorem for metric measure spaces. Anal. Geom. Metr. Spaces 3 (2015), 278-295.

[KI] B. Klartag. Needle Decompositions in Riemannian Geometry (Memoirs of the American Mathematical Society, Providence, RI, 249). 2017.

[KM] A. V. Kolesnikov and E. Milman. Brascamp-Lieb-type inequalities on weighted Riemannian manifolds with boundary. J. Geom. Anal. 27 (2017), 1680-1702.

[Ku] K. Kuwada. A probabilistic approach to the maximal diameter theorem. Math. Nachr. 286 (2013), 374-378.

[Le] M. Ledoux. The Concentration of Measure Phenomenon. American Mathematical Society, Providence, RI, 2001.

[LV] J. Lott and C. Villani. Ricci curvature for metric-measure spaces via optimal transport. Ann. of Math. (2) 169 (2009), 903-991.

[Mi] E. Milman. Beyond traditional curvature-dimension I: new model spaces for isoperimetric and concentration inequalities in negative dimension. Trans. Amer. Math. Soc. 369 (2017), 3605-3637.

[Ob] M. Obata. Certain conditions for a Riemannian manifold to be isometric with a sphere. J. Math. Soc. Japan 14 (1962), 333-340.

[Oh1] S. Ohta. Finsler interpolation inequalities. Calc. Var. Partial Differential Equations 36 (2009), 211-249.

[Oh2] S. Ohta. $(K, N)$-convexity and the curvature-dimension condition for negative $N$. J. Geom. Anal. 26 (2016), 2067-2096.

[Oh3] S. Ohta. Needle decompositions and isoperimetric inequalities in Finsler geometry. J. Math. Soc. Japan 70 (2018), 651-693.

[ON] B. O’Neill. Semi-Riemannian Geometry: With Applications to Relativity. Academic Press, New York, 1983.

[vRS] M.-K. von Renesse and K.-T. Sturm. Transport inequalities, gradient estimates, entropy and Ricci curvature. Comm. Pure Appl. Math. 58 (2005), 923-940.

[Qi] Z. Qian. Estimates for weighted volumes and applications. Q. J. Math. Oxford (2) 48 (1997), $235-242$.

[Sa1] Y. Sakurai. Rigidity of manifolds with boundary under a lower Ricci curvature bound. Osaka J. Math. 54 (2017), 85-119. 
[Sa2] Y. Sakurai. Rigidity phenomena in manifolds with boundary under a lower weighted Ricci curvature bound. Preprint, 2016, arXiv:1605.02493.

[St1] K.-T. Sturm. On the geometry of metric measure spaces. I. Acta Math. 196 (2006), 65-131.

[St2] K.-T. Sturm. On the geometry of metric measure spaces. II. Acta Math. 196 (2006), 133-177.

[Vi] C. Villani. Optimal Transport, Old and New. Springer, Berlin, 2009.

[Wy] W. Wylie. A warped product version of the Cheeger-Gromoll splitting theorem. Trans. Amer. Math. Soc. 369 (2017), 6661-6681.

Cong Hung Mai

Department of Mathematics

Kyoto University

Kyoto 606-8502

Japan

(E-mail: hongmai@math.kyoto-u.ac.jp) 